\title{
The PPAR 2 amino acid polymorphism Pro 12 Ala is prevalent in offspring of Type II diabetic patients and is associated to increased insulin sensitivity in a subgroup of obese subjects
}

\author{
M. Koch, K. Rett, E. Maerker, A. Volk, K. Haist, M. Deninger, W. Renn, H. U. Häring \\ Medical Clinic University of Tübingen, Department IV, Internal Medicine, Endocrinology, Metabolism and Pathobiochemistry, \\ 72076 Tübingen, Germany
}

\section{Abstract}

Aims/hypothesis. Recently a mutation in the coding sequence of the adipocyte specific isoform peroxisome proliferator-activated receptor $\gamma 2(\operatorname{PPAR} \gamma 2)$ was described, leading to the substitution of Proline to Alanine at codon 12. Mutations in PPAR $\gamma 2$ could have a role in people who are at increased risk for the development of obesity and Type II (non-insulindependent) diabetes mellitus.

Methods. Non-diabetic first-degree relatives $(n=108)$ of subjects with Type II diabetes were characterized by oral glucose tolerance tests and euglycaemic hyperinsulinaemic glucose clamp to determine insulin sensitivity.

Results. We found 75 (69\%) probands without the $\operatorname{PPAR} \gamma$ ProAla12 substitution, 28 heterozygotes
$(26 \%)$ and $5(4 \%)$ homozygotes. When the whole group was analysed for an association between the mutation and insulin sensitivity, no statistical significance could be shown. Only in the group with severe obesity more than $30 \mathrm{~kg} / \mathrm{m}^{2}$, an association $(p=$ $0.016)$ of the polymorphism with an increase in insulin sensitivity was found.

Conclusion/interpretation. These observations suggest that the mutation in the PPAR $\gamma 2$ molecule may have a role in subgroups prone to the development of obesity and Type II diabetes. [Diabetologia (1999) 42: 758-762]

Keywords Insulin resistance, Type II diabetes, obesity, peroxisome proliferator activated receptors, Pro 12 Ala mutation.
The peroxisome proliferator activated receptors (PPAR) are members of the nuclear hormone receptor superfamily which act as transcription factors regulating important mechanisms in adipocyte differentiation. Natural ligands of PPAR's are prostaglandin $\mathrm{J} 2$ and polyunsaturated fatty acids such as linoleic acid [1]. Several isoforms of PPAR's with different tissue distribution have been described (PPAR- $\alpha, \beta$, $\gamma 1$ and $\gamma 2$ ). The PPAR $\gamma 1$ isoform is expressed in sev-

Received: 8 January 1999 and in final revised form: 11 February 1999

Corresponding author: Dr. M. Koch, Labor Wagner und Partner, Werner-von-Siemens-Str. 10, 37077 Göttingen, Germany Abbreviations: ISR, Insulin secretion rate; MCR, metabolic clearance rate; PPAR, peroxisome proliferator activated receptors; Pro 12 Ala, Proline 12 Alanine. eral tissues whereas PPAR $\gamma 2$ is almost exclusively transcribed in adipose cells [2]. There is evidence that the $\operatorname{PPAR} \gamma$ receptor is involved in the regulation of insulin sensitivity [1] and the pathogenesis of insulin resistance.

One silent point mutation in exon 6 and a $\mathrm{CCA} \rightarrow$ GCA base exchange leading to the substitution of proline to alanine in codon 12 of exon 2 has recently been described [3]. This mutation could confer susceptibility to insulin resistance. Insulin resistance is an independent familial trait that predicts the development of Type II (non-insulin-dependent) diabetes mellitus. The genetic basis of Type II diabetes is polygenic and polyallelic and it is clinically apparent only when present in combination with environmental factors [4]. One prerequisite of genetic analysis in complex diseases is the phenotypic characterisation of people as comprehensively as possible. A history 
Table 1. Demographic and metabolic characteristics of first-degree relatives of Type II diabetic patients with the PPAR $\gamma 2$ codon Pro $^{12} \rightarrow$ Ala substitution

\begin{tabular}{|c|c|c|c|c|c|c|c|}
\hline & \multicolumn{3}{|c|}{$\begin{array}{l}\text { All probands }(n=108) \\
\text { PPAR } 2 \text { genotype }\end{array}$} & \multirow[t]{2}{*}{$p$} & \multicolumn{2}{|c|}{$\begin{array}{l}\text { BMI }>30(n=19) \\
\text { PPAR } \gamma 2 \text { genotype }\end{array}$} & \multirow[t]{2}{*}{$p$} \\
\hline & Pro/Pro & Pro/Ala & Ala/Ala & & Pro/Pro & X/ALA & \\
\hline Age (years) & $34.1 \pm 1.0$ & $36.5 \pm 1.6$ & $37 \pm 3.9$ & 0.39 & $35 \pm 2.5$ & $36 \pm 4$ & 0.85 \\
\hline BMI $\left(\mathrm{kg} / \mathrm{m}^{2}\right)$ & $25.7 \pm 0.57$ & $26.1 \pm 0.9$ & $24.0 \pm 2.2$ & 0.67 & $34 \pm 0.9$ & $33 \pm 1.4$ & 0.71 \\
\hline Body fat (\%) & $26.1 \pm 1.1$ & $24.4 \pm 1.8$ & $22.0 \pm 4.3$ & 0.52 & $38 \pm 2.4$ & $35 \pm 4.0$ & 0.47 \\
\hline Weight (kg) & $76.8 \pm 1.9$ & $78.3 \pm 2.5$ & $74.0 \pm 4.7$ & 0.82 & $101 \pm 4$ & $96 \pm 3$ & 0.48 \\
\hline Waist-to-hip ratio & $0.82 \pm 0.008$ & $0.86 \pm 0.017$ & $0.85 \pm 0.028$ & 0.09 & $0.86 \pm 0.02$ & $0.95 \pm 0.02$ & 0.08 \\
\hline $\operatorname{MCR}\left(\mathrm{ml} \cdot \mathrm{kg}^{-1} \cdot \min ^{-1}\right)$ & $7.6 \pm 0.4$ & $7.6 \pm 0.7$ & $8.5 \pm 1.7$ & 0.85 & $3.7 \pm 0.4$ & $5.6 \pm 0.6$ & $0.016^{\mathrm{a}}$ \\
\hline MCR adjusted to BMI & $8.2 \pm 0.4$ & $8.0 \pm 0.5$ & $8.2 \pm 1.2$ & 0.98 & $7.3 \pm 0.4$ & $9.0 \pm 0.5$ & $0.049^{\mathrm{a}}$ \\
\hline MCR related to lean body mass & $10.5 \pm 0.5$ & $10.0 \pm 0.7$ & $10.9 \pm 1.3$ & 0.88 & $6.1 \pm 0.5$ & $8.7 \pm 0.8$ & $0.025^{\mathrm{a}}$ \\
\hline $\begin{array}{l}\text { MCR related to lean } \mathrm{BM} \text { adjusted } \\
\text { to } \mathrm{BMI}\end{array}$ & $10.7 \pm 0.5$ & $10.4 \pm 0.6$ & $10.6 \pm 1.3$ & 0.94 & $8.8 \pm 0.6$ & $11.3 \pm 0.7$ & $0.041^{\mathrm{a}}$ \\
\hline
\end{tabular}

Data are means \pm standard error of the mean. Means of the variables were compared by analysis of variance (ANOVA) and $t$-test. In the subgroup with BMI more than $30 \mathrm{~kg} / \mathrm{m}^{2}$, het-

of Type II diabetes in first-degree relatives is associated with an increased risk for the offspring [5]. We therefore examined the prevalence of the codon proline 12 alanine (Pro12Ala) mutation in young healthy non-diabetic first-degree relatives of subjects with Type II diabetes who underwent extensive metabolic phenotyping. In contrast to unselected diabetic groups, sorting out homogenous sub-groups of subjects based on subphenotypes should increase the probability of detecting or excluding an influence of the codon Pro12Ala mutation in PPAR $\gamma 2$ on quantitative traits.

\section{Subjects and methods}

Probands. We examined 108 (48 male, 60 female) first-degree relatives of Caucasian subjects with a history of Type II diabetes. The study participants were recruited from a study launched at the department of endocrinology at the University of Tübingen for the early diagnosis in families with a history of Type II diabetes. All probands had given written consent to their participation. The study was approved by the local ethics committee and it was carried out in accordance to the Helsinki guidelines.

Methods. Insulin sensitivity was determined by euglycaemichyperinsulinaemic glucose-clamp as described elsewhere [6] and expressed as clamp-derived glucose metabolic clearance rate (MCR) expressed as virtual blood volume cleared from glucose per min and $\mathrm{kg}$ body weight $\left(\mathrm{ml} \cdot \mathrm{kg}^{-1} \cdot \mathrm{min}^{-1}\right)$. Metabolic clearance rate was also calculated in relation to lean body mass. Body composition was measured by bioelectrical impedance as percentage body fat. Glucose tolerance was determined by an oral glucose test with $40 \mathrm{~g}$ glucose $/ \mathrm{m}^{2}$ to adjust the amount of glucose given to the body weight of the probands. Determination of insulin was done with an enzyme immuno assay ( $\mathrm{IM}_{\gamma}$ Insulin, Abott Laboratories, Chicago, Ill., USA) and C peptide was measured by radioimmuno assay (RIA-coat C Peptid, Byk-Sangtec Diagnostica, Dietzenbach, erozygotes and homozygotes are united into one group. ${ }^{\text {a }}$ statistically significant differences

Germany). Body mass index was calculated as weight divided by the square of height $\left(\mathrm{kg} / \mathrm{m}^{2}\right)$. Relative rates of insulin secretion (insulin secretion rate, ISR) during OGTT were calculated from plasma insulin and $\mathrm{C}$ peptide concentrations using the algorithm described previously [7]. This method allows an estimation of the rate of appearance of newly secreted insulin.

The PPAR $\gamma 2$ Pro12Ala polymorphism was determined by PCR and subsequent restriction enzyme analysis with BstU-I [3].

For statistical analysis, differences of the means of variables were tested by $t$-tests or analysis of variance (ANOVA) if more than two groups were compared. Longitudinal data of the OGTT's were tested by multivariate analysis with time as an effect of repeated measures (MANOVA). All statistical tests were done with the JMP software (JMP Version 3.2.1, SAS Institute Inc., Cary, N.C., USA). To exclude the possibility of bias, all tests were also done on randomly choosen subgroups of the sample. All tests were two-sided and a $p$ value of less than 0.05 was considered as statistically significant.

\section{Results}

We found $75 \quad(69 \%, 95 \%$ confidence interval $61 \%-78 \%, 30$ men, 45 women) probands without the PPAR $\gamma$ Pro12Ala substitution, $28(26 \%, 95 \%$ CI $18-34 \%$ ) heterozygotes (15 men, 13 women) and 5 $(4,6 \%, 95 \%$ CI 1\%-8\%) homozygotes (3 men, 2 women). The allele frequency of the mutated allele was $17 \%$. The expected genotype distribution was in the Hardy-Weinberg equilibrium with expected frequencies of $67 \%, 28 \%$ and $3 \%$. The frequency of the mutation did not differ in probands with body mass indices above and below $30 \mathrm{~kg} / \mathrm{m}^{2}$ as judged by a contingency table analysis. Insulin sensitivity [clamp-derived glucose metabolic clearance rate $(\mathrm{MCR})]$ varied across a wide range $\left(1,65-25,2 \mathrm{ml} \cdot \mathrm{kg}^{-1} \cdot \mathrm{min}^{-1}\right)$.

Table 1 shows the results of the anthropometric and metabolic examinations. Statistical analysis by ANO- 

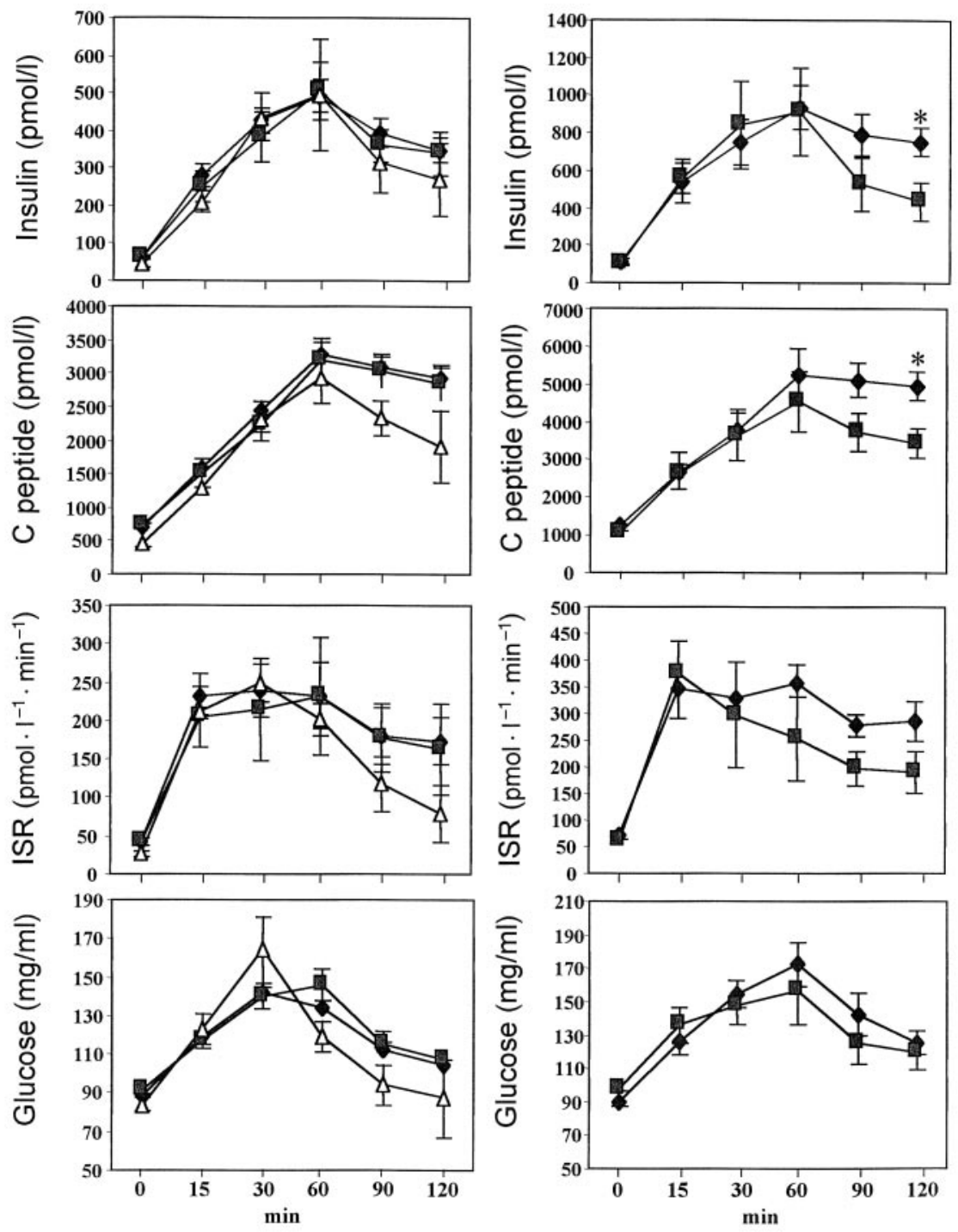

Fig. 1. Course of insulin, $\mathrm{C}$ peptide, insulin secretion rate (ISR) and glucose during oral glucose tolerance tests in 108 young, healthy first-degree relatives of Type II diabetic patients dependent on presence or absence of the PPAR $\gamma 2$ Pro12Ala mutation. Data are means \pm standard error of the mean. Longitudinal data of insulin, $\mathrm{C}$ peptide and ISR of the OGTT's were compared by multivariate analysis (MANOVA). On the left side the data of all probands are shown. The diagrams on the right show the values for the probands with a BMI more than 30 . * denotes statistically significant differences. Wildtype Pro/Pro, $\square$ heterozygous Pro/Ala $\triangle$ homozygous Ala/Ala

VA did not show significant differences in metabolic characteristics between the genotypes when the whole group was analysed. No relation between the polymorphism and waist, waist to hip ratio and lean body mass could be detected. When only probands with BMI more than $30 \mathrm{~kg} / \mathrm{m}^{2}$ were compared, a statistically significant higher MCR could be observed in probands with the mutation $(p=0.016)$. This observation

held true for MCR related to lean body mass. As body mass index was a regressor of MCR $(p<0.0001)$, BMI-adjusted MCR and BMI-adjusted MCR related to lean body mass were calculated. Both proved to be different between probands with and without the polymorphism. Significantly lower insulin $(p=0.04)$ and $C$ peptide $(p=0.025)$ concentrations in the 120-min values of oral glucose tolerance tests (Fig.1) were seen in the obese probands having the Ala allele. When analysed by multivariate analysis, which compares the mean values of the whole curves, no statistically significant differences could, however, be seen. To exclude the possibility of bias, all tests were also done on randomly choosen subgroups of the sample. The metabolic characteristics were not influenced by sex (with the exception of body fat content) in the whole group and in the subgroups. Figure 1 shows insulin, $\mathrm{C}$ peptide, insulin secretion rates and glucose concentrations of all probands (Fig. 1 left) and the obese subgroup (Fig. 1 right) comparing probands with and without the Ala allele. 


\section{Discussion}

Type II diabetes develops over a long period from an interplay between insulin resistance, a relative deficiency of insulin secretion capacity and environmental factors. The defects are detectable when the patients are normoglycaemic and in most cases more than a decade before diagnosis of the disease [8]. Insulin resistance as part of the metabolic syndrome and precursor of overt Type II diabetes has a genetic but as yet unknown basis [9]. Disturbances in the regulation of adipogenesis are a well known feature in Type II diabetic patients. One of the key elements in adipocyte development is PPAR $\gamma 2$, a transcription factor of the nuclear hormone receptor superfamily with profound influences in adipocyte differentiation, adipogenesis, gene expression and insulin action.

First-degree relatives of Type II diabetic patients have a $40 \%$ risk of developing diabetes [8] in contrast to the overall $4 \%$ risk in the general population. We therefore investigated a group of young, healthy first-degree relatives for the presence of the Pro12Ala polymorphism and possible influences on body mass index, percentage body fat, insulin sensitivity as measured by euglycaemic hyperinsulinaemic clamp and insulin secretion.

The PPAR $\gamma 2$ Pro12Ala polymorphism was present in our group of first-degree relatives of patients with Type II diabetes with a frequency of $26 \%$ heterozygous, around $5 \%$ homozygous probands and an allele frequency of 0.17 . An association with BMI could not be seen in our group. From 19 probands with a BMI more than $30 \mathrm{~kg} / \mathrm{m}^{2} 5$ had the mutation and 28 from 89 with BMI less than $30 \mathrm{~kg} / \mathrm{m}^{2}$ $(p=0.65)$. In general, homozygous (Ala/Ala) probands had lower insulin, $\mathrm{C}$ peptide, insulin secretion rate and glucose concentrations in the late phase of OGTT's (Fig.1, left), but the differences did not reach statistical siginificance.

In severely obese subjects $\left(\mathrm{BMI}>30 \mathrm{~kg} / \mathrm{m}^{2}\right)$ an association of the PPAR $\gamma 2$ Pro12Ala polymorphism with increased insulin sensitivity could be shown. Compared to insulin sensitivity in all probands (MCR $7.6 \mathrm{ml} \cdot \mathrm{kg}^{-1} \cdot \mathrm{min}^{-1}$ ), the obese group had a lower insulin sensitivity (MCR $4.3 \mathrm{ml} \cdot \mathrm{kg}^{-1} \cdot \mathrm{min}^{-1}$ ) but within the obese group, the Pro12Ala mutation was associated with an increase in insulin sensitivity (3.7 vs $5.6 \mathrm{ml} \cdot \mathrm{kg}^{-1} \cdot \mathrm{min}^{-1}, p=0.016$ ). Lower insulin concentrations, $\mathrm{C}$ peptide and insulin secretion rates could be seen in the OGTT in those probands who bear the Ala allele. Significant differences in C peptide and insulin concentrations were restricted to the 120 min values in the OGTT ( $p=0.025$ and $p=0.04$, repectively). This would suggest an effect of the mutation for the late phase of insulin secretion at least in overweight subjects. As the low number of obese subjects with the Pro12Ala mutation could lead to misleading statistical results, further studies are re- quired to confirm the results and to characterize the functional mode of action of the Pro12Ala mutation in severly obese subjects. Recently, another mutation resulting in the conversion of amino acid proline 115 to glutamine in PPAR $\gamma 2$ was described in 4 of 121 obese subjects [10]. This mutation was shown to be functionally relevant, leading to a defect in phosphorylation of serine 114 and to accelerated differentiation of cells into adipocytes and increased accumulation of triglycerides. Moreover, the mean fasting serum insulin concentration was lower $(p<0.05)$ in the four obese subjects with the mutation than in the rest of the obese subjects, suggesting a lower level of insulin resistance.

Although the functional relevance of the Pro12Ala mutation in the PPAR $\gamma 2$ gene still remains to be determined, it was shown that insulin enhances the transcriptional effect mediated by PPAR $\gamma 2$ by activating a ligand-independent domain in the $\mathrm{N}$ terminus of the molecule [11]. The Pro12Ala amino acid substitution is situated in the $\mathrm{N}$ terminal region which could be the functional basis of our observation that the mutation is associated with increased insulin sensitivity and lower insulin and $\mathrm{C}$ peptide concentrations in the late phase of oral glucose tolerance tests in our obese probands.

In summary, these observations show that activating mutations of PPAR $\gamma 2$ might exist which lead to obesity associated with less insulin resistance than expected [10]. The Pro12Ala mutation in the PPAR $\gamma 2$ molecule could play a part in subgroups of people prone to the development of Type II diabetes. The effects can be detected in well characterised subgroups whereas in unselected groups of people such influences could easily be missed.

Acknowledgements. This study was supported by the European BIOMED 2 Programme 'Building of a European Task Force for the Identification of Type II diabetes Susceptibility Genes in the Insulin Action Cascade' BMH4-CT96-0751.

\section{References}

1. Spiegelman BM (1998) PPAR-gamma: adipogenic regulator and thiazolidinedione receptor. Diabetes 47: 507-514

2. Auboeuf D, Rieusset J, Fajas L et al. (1997) Tissue distribution and quantification of the expression of mRNAs of peroxisome proliferator-activated receptors and liver $\mathrm{X}$ receptor-alpha in humans: no alteration in adipose tissue of obese and NIDDM patients. Diabetes 48: 1319-1327

3. Yen CJ, Beamer BA, Negri C et al. (1997) Molecular scanning of the human peroxisome proliferator activated receptor gamma (hPPAR gamma) gene in diabetic Caucasians: identification of a Pro12Ala PPAR gamma 2 missense mutation. Biochem Biophys Res Commun 241: 270-274

4. Kahn CR (1995) Diabetes. Causes of insulin resistance. Nature 373: 384-385

5. Eriksson J, Franssila Kallunki A, Ekstrand A et al. (1989) Early metabolic defects in persons at increased risk for 
non-insulin-dependent diabetes mellitus. N Engl J Med 321: 337-343

6. DeFronzo RA, Tobin JD, Andres R (1979) Glucose clamp technique: a method for quantifying insulin secretion and resistance. Am J Physiol 237:E214-E223

7. Polonsky KS, Licinio-Paixao J, Given Bd et al. (1986) Use of biosynthetic human C-peptide in the measurement of insulin secretion rates in normal volunteers and type I diabetic patients. J Clin Invest 77: 98-105

8. Martin BC, Warram JH, Krolewski AS, Bergman RN, Soeldner JS, Kahn CR (1992) Role of glucose and insulin resistance in development of type 2 diabetes mellitus: results of a 25-year follow-up study. Lancet 340: 925-929
9. Reaven GM (1995) Pathophysiology of insulin resistance in human disease. Physiol Rev 75: 473-486

10. Ristow M, Müller-Wieland D, Pfeiffer A, Krone W, and Kahn CR (1998) Obesity associated with a mutation in a genetic regulator of adipocyte differentiation. N Engl J Med 339(14):953-959

11. Werman A, Hollenberg A, Solanes G, Bjorbaek C, VidalPuig AJ, Flier JS (1998) Ligand-independent activation domain in the $\mathrm{N}$ terminus of peroxisome proliferator-activated receptor gamma (PPARgamma). Differential activity of PPARgamma1 and -2 isoforms and influence of insulin. J Biol Chem 272: 20230-20235 